\title{
FACTORES DE ABANDONO ESTUDIANTIL EN LA CARRERA DE INGENIERÍA EN ZOOTECNIA, RECINTO NUEVA GUINEA
}

MSc. Arsenio López Borge MSc. Napoleón Rojas Robles ${ }^{[4]}$

\section{Resumen}

El objetivo de este trabajo fue en identificar los factores de incidencia en el abandono de la carrera de Ingeniería en Zootecnia, la URACCAN, Recinto Nueva Guinea. El estudio es descriptivo, incursiona en diferentes tópicos torales, por medio de los cuales se buscaron las respuestas con el fin de explicar los factores que incidieron de manera significativa en el abandono de la carrera por parte de los estudiantes. Presenta de forma explícita las causas de la deserción. Los resultados establecen que éstas tienen correspondencia con las actividades curriculares y extracurriculares en el ámbito universitario. El estudio al profundizar en la temática de la deserción, aborda el desempeño estudiantil en sus distintas esferas y dimensiones: socio - económicas y académicas, de igual manera el ejercicio docente y los aportes metodológicos para el aprendizaje, concluyendo que las principales causales de éstas se reflejan en los ámbitos: socio - económico, el subsistema universitario, académico, personal en la falta de conocimiento del perfil de la carrera y la falta de motivación, incluye la incompleta preparación del bachillerato para enfrentar los retos de la Educación Terciaria.

Palabras Clave: deserción, abandono, enseñanza, aprendizaje, docencia, factores.

\section{Introducción}

La deserción escolar es un problema que afecta a la mayoría de las instituciones de Educación Superior del país, para el desarrollo social y económico de cualquier nación. En diez países de América latina, en el período 2000 - 2005, se presenta una media aritmética de abandono escolar del 62.8 por ciento. En Nicaragua, se representa el porcentaje más bajo de deserción con el 40 por ciento, en comparación con república Dominicana (90 \%) y Bolivia (95\%), quienes tienen los índices más altos (González, 2005).

De estos datos se infiere la necesidad de buscar causas y factores que inciden en la permanencia del problema y en la búsqueda de soluciones que procuren disminuir la deserción temprana en los niveles de Educación Primaria y Secundaria. Del anterior

[4] Docentes de la Universidad de las Regiones Autónomas de la Costa Caribe Nicaragüense (URACCAN). 
nivel, de los que ingresan a la Educación Terciaria: "Sólo el 2.46 por ciento culminan sus estudios en el tiempo corriente” (Sandino, 2006).

En Nicaragua los estudiantes universitarios dejan sus estudios por limitaciones económicas, aunque otros lo hacen porque no pueden cumplir las exigencias académicas y el abandono escolar oscila entre un 30 y 40 por ciento (González, 2005). Miles de universitarios tienen que abandonar la universidad todos los años.

En la URACCAN donde mayormente incide el fenómeno de la deserción es en la carrera de Ingeniería en Zootecnia, que se oferta en dos recintos (Siuna y Nueva Guinea), en los cuales, según información de los registros académicos, la deserción o abandono ocurre en los primeros años de estudios.

La Investigación fue necesaria, porque los datos estadísticos reflejan una deserción promedio del 40 por ciento. Cuestión fundamental que ha de servir de base para que las autoridades competentes diseñen políticas educativas y académicas orientadas hacia la reducción de este fenómeno escolar hasta reducirla a los niveles mínimos aceptables.

\section{Marco Teórico}

\section{Deserción Escolar como problema en el sistema educativo}

La deserción escolar se refiere a la masa de estudiantes que abandonan sus estudios en cualquier momento del año escolar y se presenta en todas las escuelas ocasionando problemas para el desarrollo del proceso educativo, por ello es considerada como una de las causas del proceso escolar que requiere ser abordada en forma efectiva y sistemática, siendo este un problema de índole psicosocial que modifica la conducta del educando en relación a su presencia en el sistema educativo (Goicovic, 2002).

\section{Culminación del proceso educativo y deserción escolar}

Cuando culmina el proceso educativo y se presentaron problemas de deserción la institución escolar tiene una alta cuota de responsabilidad, así como las autoridades, el estudiante y su familia, aún así el Ministerio de Educación tiene claramente establecido los fines que persigue en el desarrollo del proceso educativo en Nicaragua y entre ellos el de formar hombres y mujeres con conocimientos científicos, técnicos, culturales, humanísticos e históricos (Mined., 2007).

\section{Deserción escolar en América Latina}

Las altas tasas de deserción escolar registrada en la gran mayoría de países de Ámerica Latina se traducen en un bajo número de años de educación aprobados, muy por debajo del ciclo secundario completo, considerado como el capital educativo mínimo 
necesario para insertarse en empleos urbanos con altas posibilidades de situarse fuera de la pobreza, en estos países en la zona urbana las niñas abandonan la escuela con menos frecuencia que los niños y además, muestran un menor índice de repetición que les permite completar la Educación Secundaria sin retraso. En cambio, en las zonas rurales las mujeres tienden a dejar de estudiar antes que los hombres, sobre todo en los primeros años de la primaria (CEPAL., 2002).

\section{Materiales y métodos}

\section{Ubicación y tipo de estudio}

El trabajo de investigación se desarrolló en el sector urbano y rural del municipio de Nueva Guinea (RAAS) y se auxilió con la realización de encuestas y entrevistas dirigidas a estudiantes y docentes del recinto universitario que trabajan para la URACCAN; la población en estudio fueron los estudiantes que han abandonado la carrera y el personal docente que laboran en las áreas relacionadas con la carrera, sin distinción alguna, la que totaliza 151.

\section{Tipo de investigación}

La investigación es de tipo descriptivo, según (Zamarrita 2007), los estudios descriptivos buscan especificar las propiedades, las características y los perfiles importantes de personas, grupos, comunidades o cualquier otro fenómeno que se someta a un análisis. Miden, evalúan o recolectan datos sobre diversos aspectos, dimensiones o componentes del fenómeno a investigar. Desde el punto de vista científico, describir es recolectar datos (para los investigadores cuantitativos, medir; y para los cualitativos, recolectar información).

\section{Universo}

El universo del estudio fue todos los estudiantes que abandonaron la carrera de Zootecnia, y los docentes que imparten asignaturas, en ésta se incluye al coordinador.

\section{Población y muestra}

Para obtener una información eficaz se realizó un muestreo estratificado considerando dos estratos: $\mathrm{El}$ estrato dos está formado por estudiantes que abandonan la carrera en el primer año de estudio, y el estrato uno por aquellos que abandonan sus estudios en segundo y tercer año.

El tamaño de la muestra se calculó mediante el empleo de la fórmula que define el número de muestra "n" para poblaciones conocidas: 


$$
n=\frac{p^{*} q}{\frac{E^{2}}{z^{2}}+\frac{p^{*} q}{N}}
$$

Donde:

$\mathrm{n}=$ Tamaño de la muestra

$\mathrm{p}=$ probabilidad de ocurrencia de un evento

$\mathrm{q}=$ probabilidad de no ocurrencia de un evento

$\mathrm{E}=$ Margen de error

$Z=$ Nivel de confianza

$\mathrm{N}=$ Población de interés

Para esta investigación se estableció un nivel de confianza del 95 por ciento $(Z=$ 1.96), un margen de error del 4 por ciento, la probabilidad de ocurrencia o no ocurrencia sumará la unidad (que representa el $100 \%$ ) y utilizando la población de interés definida anteriormente y se determinó que el tamaño de la muestra será de 50 individuos

$$
n=\frac{(0.6)(0.4)}{\left(\frac{0.04^{2}}{1.96^{2}}\right)+\frac{(0.6)(0.5)}{57}}=
$$

$$
\frac{0.24}{\left(\frac{0.0016}{3.8416}\right)+\left(\frac{0.24}{57}\right)}=\frac{0.24}{0.000416493+0.004210}=\frac{0.24}{0.00462664}=51.8
$$

Se ajusta la muestra a cincuenta (50) que es una cantidad probalística de la población de interés.

Para la estratificación se usó la formula siguiente Ksh $=\mathrm{n} / \mathrm{N}$; que es la fórmula que se usa para estratificar, de la siguiente manera: $(50 / 57=0.8741)$

La muestra del estrato uno es: $(0.8741 * 25=22)$ 
La muestra del estrato dos es: $\left(0.8741^{*} 32=28\right)$

\section{Criterios de exclusión}

Para este estudio no fueron parte de la investigación los estudiantes activos y egresados de la carrera de Ingeniería en Zootecnia así como los estudiantes de las otras disciplinas del recinto universitario URACCAN, Nueva Guinea.

También hay que hacer mención que se excluyeron los estudiantes de la carrera de zootecnia que han abandonado sus estudios en los otros recintos universitarios y extensiones.

\section{Delimitación y limitaciones del estudio}

Este estudio fue realizado únicamente en el recinto universitario de Nueva Guinea RAAS, Nicaragua; específicamente con estudiantes de la carrera de Zootecnia que abandonaron el sistema escolar en los periodos del 2004 al 2006.

Las limitantes para que la investigación no se realizara son que los estudiantes, fue que estos no se encontrarán en el municipio, o que no se tuviera un registro de los nombres de los estudiantes, o que se rehusaron a contestar.

\section{Técnicas e instrumentos}

Las técnicas utilizadas para la obtención de información fueron a través de encuestas estructuradas y entrevistas a estudiantes que abandonaron el sistema escolar, incluyendo a docentes activos en el recinto tanto horarios como permanentes y los instrumentos a utilizados fueron los cuestionarios, guías de campo, listado de estudiantes que abandonaron el sistema escolar.

\section{Resultados y discusión}

El gráfico No. 1, representa la consulta realizada en el semestre que los estudiantes abandonaron la carrera y en la cual se constata que el 80 por ciento (40 estudiantes) se alejaron de la vida universitaria en los primeros dos años de estudio y el mayor índice de deserción se da en el primer año de la carrera, (50\%) por lo tanto, la atención para lograr mayor retención se debe orientar hacia los dos primeros semestres. 


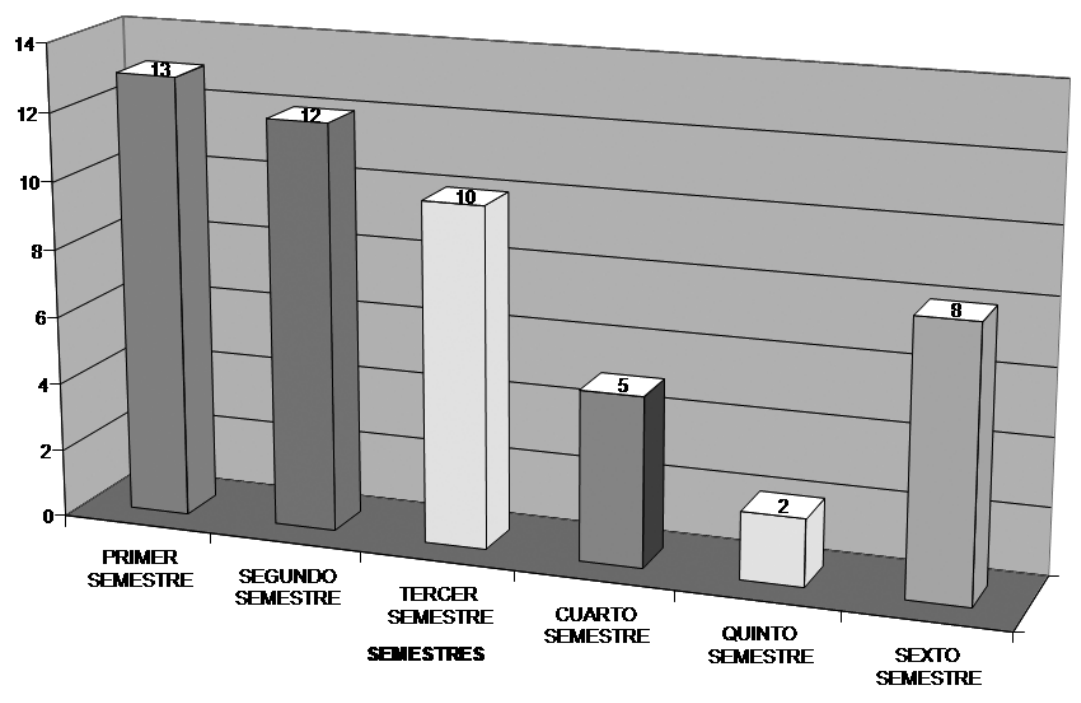

Gráfico No. 1. Semestre en que abandonó la universidad

Los resultados obtenidos en relación a la variable: quien pagaba los estudios (gráfico No. 2), se presenta una contradicción; no obstante, la misma se justifica en el hecho de que los estudiantes que abandonaron la carrera consideran que el sostén de los estudios, ya sea por sus padres, familiares o por el usufructo de medios de producción familiar es una sola condición, de esta forma se considera que el 98 por ciento de los gastos incurridos en la formación profesional son asumidos por la familia, además, es necesario señalar que esta parte se refiere a costos de alimentación, transporte y estancia, exceptuando así, los costos de aranceles (matrícula y pago por semestre).

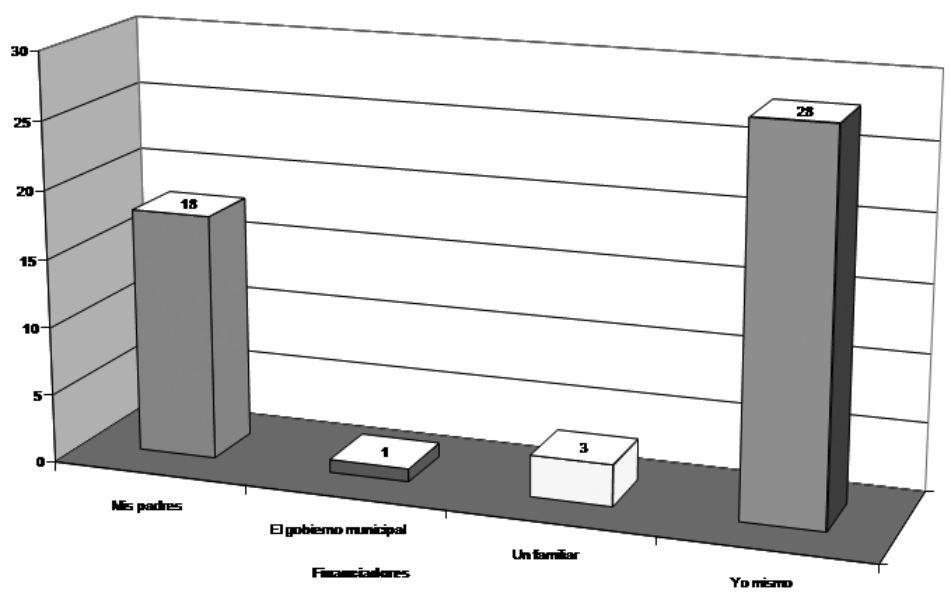

Gráfico No. 2: quien financiaba los estudios 
El gráfico No. 3, presenta una situación común en nuestro país y en particular en la región, los bachilleres en su mayoría no reciben orientación vocacional acerca de la carrera a elegir, por ello los resultados de este estudio no son una excepción dado que el 70 por ciento optó por la carrera de zootecnia, sin conocer concretamente en qué expedición estaban incursionando. La diferencia el 30 por ciento tenía nociones básicas sobre lo que pretendían estudiar las cuales fueron sustentadas a través de amigos, docentes y padres de familia.

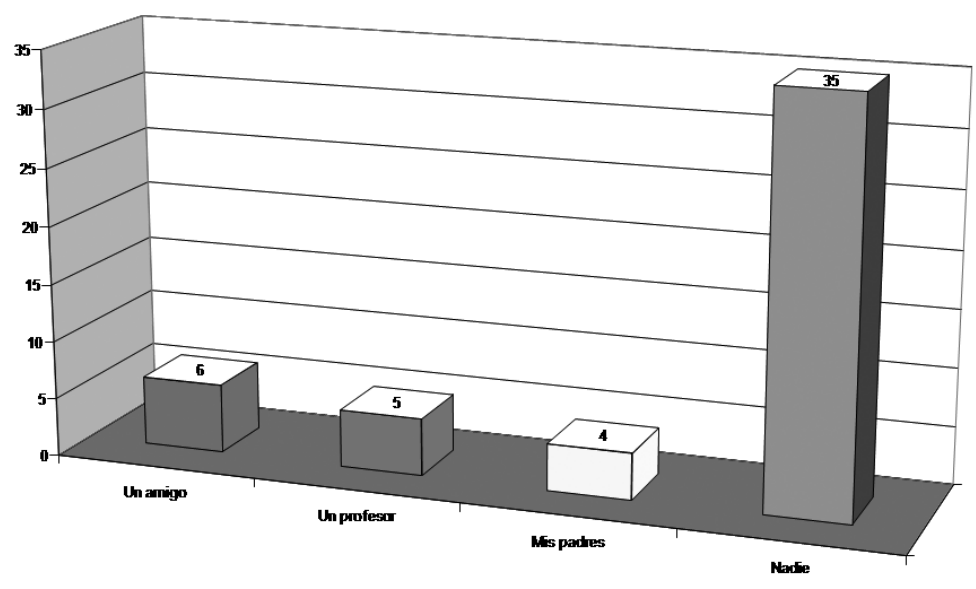

Gráfico No. 3: De quien recibió orientación vocacional

Sobre los planes que tienen los estudiantes que abandonaron la carrera (gráfico No. 4) subjetivamente el 56 por ciento pretende continuar sus estudios universitarios, de ellos un 6 por ciento está matriculado en otra universidad, un 2 por ciento tiene planes de estudiar en otra universidad y el 48 por ciento pretende regresar a URACCAN y finalmente el 44 por ciento restante está indeciso o no tiene proyecto inmediato para estudiar. Estos resultados indican que aproximadamente un 50 por ciento de los que dejaron la carrera pretenden reingresar a URACCAN a continuar sus estudios pudiendo optar por una nueva carrera, lo que indica que hay confianza en esta casa de estudios superiores. 


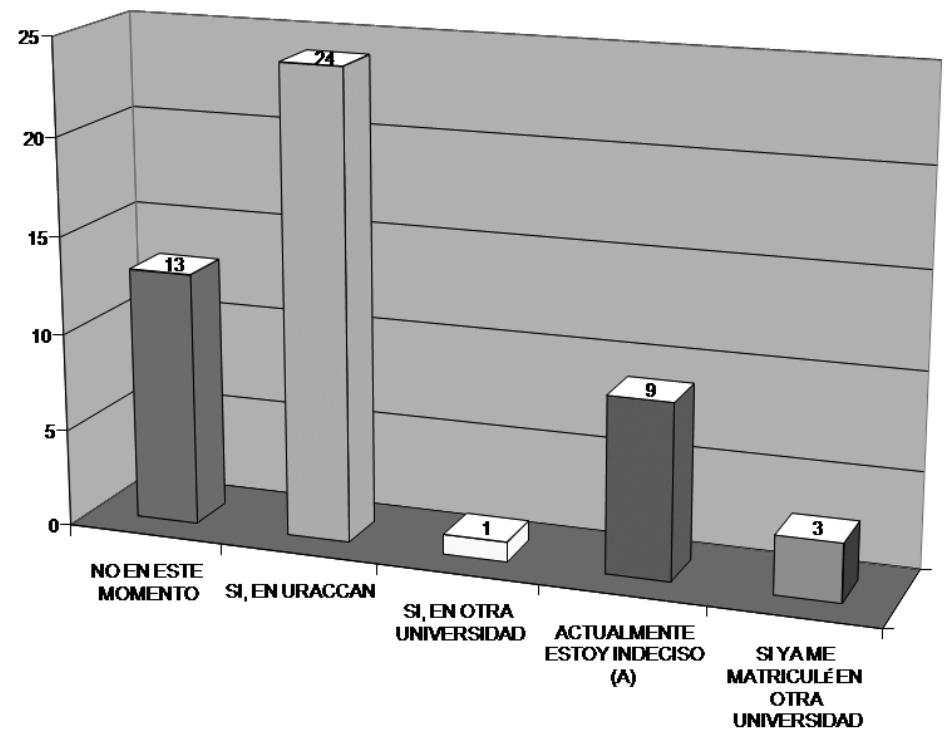

Gráfico No. 4: Planes de seguir estudiando

Lo reflejado por el gráfico No. 5 , indica de que los costos de la carrera en URACCAN no fue un factor para que abandonasen la misma, ya que el 98 por ciento de los consultados expresan que la inversión fluctuaba entre costeable, accesible y muy accesible; un 2 por ciento opinó que era apenas costeable, lo que no es significativo para este estudio.

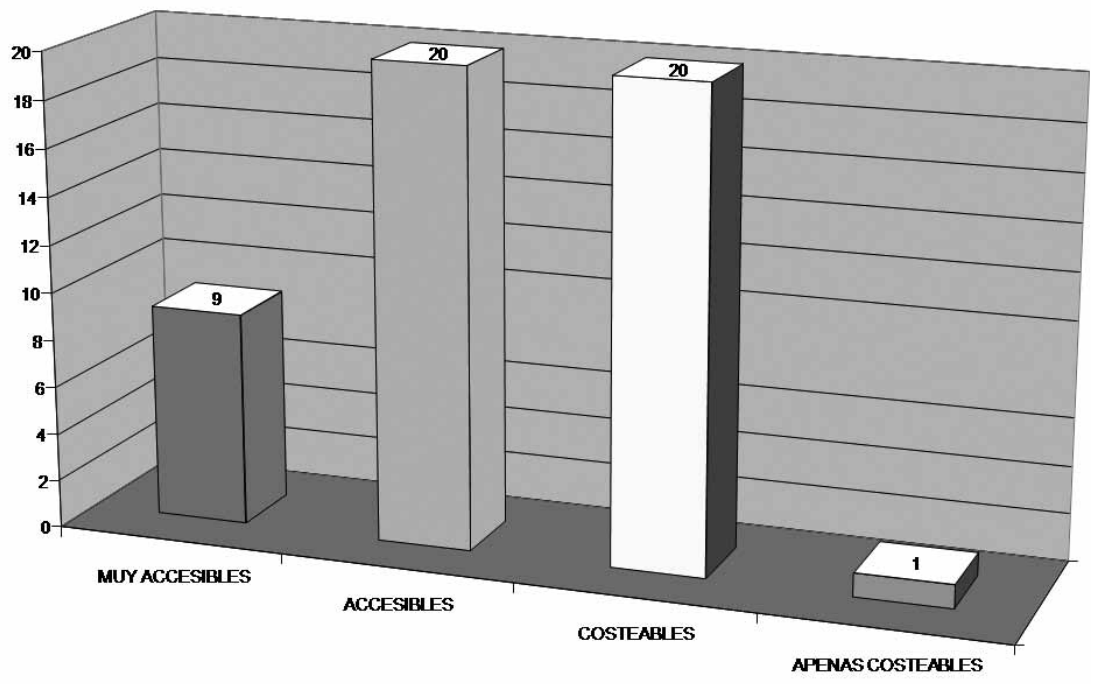

Gráfico No. 5: Costos de la carrera 


\section{Resultados de entrevistas aplicadas a docentes de la carrera de ingeniería en zootecnia.}

Con el objetivo de obtener la percepción, aportes y criterios de los docentes acerca de la deserción escolar en la carrera de zootecnia, se les realizó entrevistas a diez (10) docentes de los veinte (20) que laboran en la carrera para un tamaño de muestra del 50 por ciento.

Un 5 por ciento de los entrevistados le atribuyen al abandono escolar al perfil de la carrera el cual expresan, que los programas están desfasados y cargados en unidades difíciles de desarrollar en relación al tiempo, consideran que deben de mejorarse.

Cabe hacer mención que existen causas de mayor peso por parte de los estudiantes el cual se sale de las manos de las autoridades de la Universidad resolver.

A continuación se indican las causas más sobresalientes del abandono escolar desde la percepción de los docentes.

\section{Factor económico}

- Trato no acorde con la ética profesional hacia los estudiantes por algunos docentes.

- Falta de conocimiento del perfil de la carrera.

- Motivación insuficiente de parte de los docentes.

- Programas desfasados.

- $\quad$ El pensum muy cargado.

\section{Conclusiones}

Las causas o factores que tiene mayor peso para que los jóvenes del recinto URACCAN Nueva Guinea abandonen sus estudios de licenciatura se identificaron en cuatro ámbitos: Socioeconómico, del propio sistema Universitario, de orden académico, y personales.

1. Para que un estudiante abandone una carrera universitaria se combinan aspectos como el lugar donde reside, el nivel de ingreso, el nivel educativo de los padres de familia, la necesidad de trabajar para mantenerse o contribuir a los ingresos familiares.

2. La investigación indica que los estudiantes se sienten motivados para regresar al sistema educativo porque el hecho de no tener estudio hace que a muchas personas les paguen menos por su trabajo, así lo realicen igual que otra persona que estudió; también cuando los hijos e hijas de estas personas crecen y tienen más 
estudio que ellas, creen que son mejores que sus padres y madres, entonces se da una presión en los adultos y en las adultas para ponerse al nivel de la familia y mantener su estatus.

3. Otro motivo cultural que tienen los jóvenes para reingresar es que se dan cuenta que a menos cantidad de estudio se les cierran las posibilidades para ingresar a otros espacios que tal vez les brindarían más satisfacciones.

4. La mayoría de las personas que participaron en las entrevistas quieren seguir estudiando una carrera técnica o universitaria, es decir no ven a la Educación Formal como un fin, sino como un paso para ingresar a otros espacios institucionales.

5. De la investigación se finiquita que en el Sistema Escolar no existen, políticas de retención de los y las estudiantes en el Sistema Educativo, ni hay seguimiento de lo que pasa con los y las estudiantes que desertan

6. También concluimos con esta investigación que los y las dicentes que presentan problemas de comportamiento o académico son empujados de una Institución a otra durante su permanencia en el Sistema Escolar y esto lleva a que muchas veces deserten, pues, no se cuenta con mecanismos para darles una atención diferente a la que se da para la mayoría de la población estudiantil y por ello al no existir políticas de retención, sino soluciones parciales como: Alimentación, medias becas, continúa la deserción y el que muchas personas se conviertan en minoría por falta de estudio.

\section{Lista de referencias}

Asamblea Nacional. (2003). Constitución Política de la República de Nicaragua con sus reformas. 9na Edición. Editorial Jurídica. Managua, Niacaragua.

CEPAL. (2002). Panorama social de América Latina, 2001- 2002(LC/G.2183-P/E), Santiago de chile. Publicación de las naciones unidas.

Espíndola, E. y León, A. (2003). La deserción escolar en América Latina. OEI. Revista iberoamericana No 30 (Septiembre - Diciembre 2002).

Freire, P. (1997). Pedagogía de la autonomía. Saberes necesarios en la práctica educativa. Río de Janeiro: Paz Terra.

Galeana, Rosaura. (1997). La infancia desertora. SNTE- MEXICO. Revista La educación en nuestras manos No 64. julio - Agosto -2001).

Gargiulo, C. y Crouch, L.A. (1994). Nicaragua: escolaridad, repetición y deserción escolar. Resultados de una encuesta nacional. (Borrador preliminar), Managua, Proyecto ABEL, USAID. 
Gaviria, A, Uribe, A. (2006). Antioquia se toma la palabra. Deserción escolar. Editorial Colmbia.

Goicovic D. (2002). Educación, deserción escolar e integración laboral juvenil. Última década, No 10. Viña del mar, ediciones CIDPA.

Herrera, M. E. (1999). Fracaso escolar, código y disciplina: una aproximación etnográfica. en. Última década No 10. Viña del mar, ediciones CIDPA.

LEY No. 582. (2006). Ley General de Educación Nicaragua.

Ministerio de Educación, Cultura y Deportes. Plan Común de Trabajo del MECD Prioridades Estratégicas para el período 2005 - 2008. Managua, 2004.

Ministerio de Educación, Cultura y Deportes. Plan Nacional de Educación 2001-2015. Managua, 2001.

ODACAN. (2003). Ley 28. Estatuto de Autonomía de las dos Regiones de la Costa Atlántica de Nicaragua y su Reglamento. 